\title{
Robot-assisted laparoscopic Nissen fundoplication using the DaVinci Xi
}

\author{
David S. Demos, William B. Tisol
}

Aurora St. Luke's Medical Center, Milwaukee, Wisconsin, USA

Contributions: (I) Conception and design: Both authors; (II) Administrative support: Both authors; (III) Provision of study materials or patients: Both authors; (IV) Collection and assembly of data: Both authors; (V) Data analysis and interpretation: Both authors; (VI) Manuscript writing: Both authors; (VII) Final approval of manuscript: Both authors.

Correspondence to: David S. Demos, MD. Aurora St. Luke's Medical Center, 2901 W. Kinnickinnic River Pkwy, Professional Office Bldg 1, Suite 507, Milwaukee, Wisconsin, USA. Email: ddemosmd@outlook.com.

\begin{abstract}
Anti-reflux surgery remains an important option for treatment of gastroesophageal reflux disease (GERD). Approaches include transthoracic and transabdominal techniques, both open and minimally invasive. Transthoracic approaches are uncommonly performed, generally being limited to use in patients with a hostile abdomen. Transabdominal 360-degree posterior wrap, or Nissen fundoplication, is the most commonly performed anti-reflux operation worldwide. The use of the Da Vinci Xi surgical robot (Intuitive Surgical, Sunnyvale, CA, USA) adds some distinct advantages to the laparoscopic approach. These include: (I) a steady camera with high-definition, stereotactic view, (II) tremor reduction through the robotic console, (III) wristed instruments providing flexibility in angle of approach and dexterity, and (IV) bi-polar energy use for more delicate, precise tissue division without excessive thermal spread. The advantage of bi-polar energy delivery over monopolar cautery cannot be overstated. We describe our approach to DaVinci Xi Robotassisted laparoscopic Nissen fundoplication.
\end{abstract}

Keywords: Gastroesophageal reflux disease (GERD); robotic laparoscopy; Nissen fundoplication

Received: 06 December 2019; Accepted: 30 January 2020; Published: 20 April 2021.

doi: 10.21037 /jovs.2020.02.04

View this article at: http://dx.doi.org/10.21037/jovs.2020.02.04

\section{Introduction}

Anti-reflux surgery remains an important option for treatment of gastroesophageal reflux disease (GERD). Approaches include transthoracic and transabdominal techniques, both open and minimally invasive. Transthoracic approaches are uncommonly performed, generally being limited to use in patients with a hostile abdomen. Transabdominal 360-degree posterior wrap, or Nissen fundoplication, is the most commonly performed anti-reflux operation worldwide, with laparoscopy being the standard approach (1). The use of the Da Vinci Xi surgical robot (Intuitive Surgical, Sunnyvale, CA, USA) adds some distinct advantages to the laparoscopic approach. These include: (I) a steady camera with high-definition, stereotactic view, (II) tremor reduction through the robotic console, (III) wristed instruments providing flexibility in angle of approach and dexterity, and (IV) bi-polar energy use for more delicate, precise tissue division without excessive thermal spread. The advantage of bi-polar energy delivery over monopolar cautery cannot be overstated. While there are no clear advantages published in the literature $(2,3)$, as experience with robotic surgery expands, robotic technique and efficiency will continue to improve and perhaps translate to superior outcomes. We describe our approach to DaVinci Xi Robot-assisted laparoscopic Nissen fundoplication (Video 1).

\section{Anesthesia and positioning}

General anesthesia is induced with single-lumen orotracheal intubation. Only peripheral intravenous access is needed. Central access, if necessary, for any reason, can 
be established in the internal jugular vein or either groin. Arterial line placement is not routinely necessary. The patient is placed supine with both arms tucked.

\section{Port placement and docking}

Port placement for this procedure is generally acceptable for all foregut operations. It utilizes all $8 \mathrm{~mm}$ ports, essentially in the same transverse plane. We prefer blunt needle abdominal insufflation just below the left costal margin. A camera port is placed two fingerbreadths superior and left of the umbilicus. This can be adjusted for patients with very long torsos. Next, the right subcostal retraction arm port is placed in the same transverse plane as far laterally as is safe. An additional working arm port is placed splitting the difference between the first two. The camera is then placed in one of the right-sided ports to allow clear visualization of the left side. A left subcostal port is placed as far laterally as is safe, again in the same transverse plane as the prior three ports. An $11 \mathrm{~mm}$ assistant port is then placed, again splitting the difference between the camera and left subcostal port.

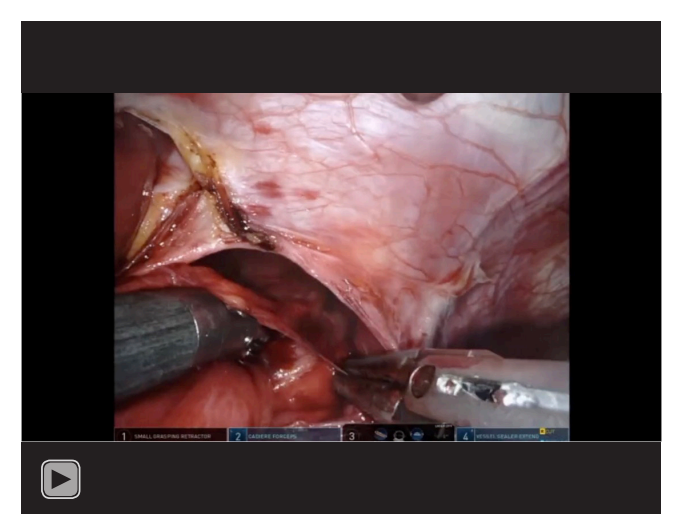

Video 1 Conduct of the robot-assisted laparoscopic Nissen fundoplication.
The patient is placed in slight reverse Trendelenburg and the robot is then brought into place for docking. The instruments are inserted under direct vision (Table 1).

\section{Technique}

The small grasping retractor in arm 1 is placed under the liver and the left lobe of the liver is retracted anteriorly with the jaws open to allow for broader exposure of the hiatus. The cadiere is then used in arm 2 along with the long bipolar in arm 4. First the gastrohepatic ligament is opened widely and the right diaphragmatic crus is exposed. The bedside assistant places an open grasper on the lesser curve of the stomach and retracts left and anteriorly. This allows dissection posteriorly to the crural decussation. The peritoneum is opened along the right crus and followed anteriorly. The bedside assistant grasps the stomach and retracts posteriorly and caudally. The surface of the esophagus is identified and slightly separated from the hiatus to facilitate identification of the gastroesophageal junction. The dissection is carried left and posteriorly along the left crus. The bedside assistant grasps the fundus of the stomach and retracts it to the right. Once the left crus is thoroughly cleared of peritoneum and retroperitoneal fat and connective tissue, the bedside assistant again retracts the stomach left and anteriorly with an open grasper to expose the crural decussation. The left crus is identified and then followed posterior to the GEJ on the abdominal side until the dissections are connected. This space is then opened widely to accommodate the fundoplication.

The gastroesophageal junction (GEJ) fat pad is elevated starting from the cardia of the stomach. This is facilitated by having the bedside assistant retract the fundus, first to the left to expose the gastric portion of the fat pad. Once the gastric portion of the fat pad is elevated off the stomach and the angle of His is identified, the bedside assistant retracts the fundus more toward the right. The

Table 1 Port and instrument placement

\begin{tabular}{lllll}
\hline Port & Size $(\mathrm{mm})$ & Location & Hand & Instrument \\
\hline Arm 1 & 8 & Right subcostal & Left & Small grasping retractor \\
Arm 2 & 8 & Right side between Arms 1 and 3 & Left & Cadiere \\
Arm 3 & 8 & Two fingerbreadths superior and left of umbilicus & & Camera \\
Arm 4 & 8 & Left subcostal & Right & Long bipolar forceps; Vessel Sealer \\
Assistant port & 11 & Left side between Arms 3 and 4 & Assistant & Suction or laparoscopic grasper \\
\hline
\end{tabular}


fat pad can then be elevated off the GEJ from left to right, staying on the esophageal wall to avoid injury to the anterior vagus nerve and mobilize it off the esophagus to facilitate the fundoplication. The GEJ is identified where the longitudinal esophageal muscle fibers fan out. The amount of intraabdominal esophagus is measured from the hiatus to this point. Measurement can be facilitated by marking the long laparoscopic suction device with a marker every $1 \mathrm{~cm}$ from the tip. If the intraabdominal length is too short, a Penrose drain can be wrapped around the GEJ for retraction, and mediastinal dissection will be necessary to free additional esophageal length.

Next, the short gastric vessels are divided. The vessel sealer is placed in arm 4 (right hand). A point midway along the fundus is chosen and the omentum is divided until the lesser sac is encountered. The retraction strategy here sometimes involves using the small grasping retractor in arm 1 to retract the stomach anteriorly and to the right, adjusting more proximally as the dissection proceeds. The omentum is then divided proximally along the fundus, through the short gastric arteries. The bedside assistant is typically most helpful here by retracting the omentum to the left. Once the short gastric vessels are divided and this dissection connects with the hiatus dissection, the fundus is completely mobile.

The small grasping retractor in arm 1 is returned to the left lobe of the liver. The stomach is again retracted to the left with an open grasper by the bedside assistant and the hiatus is assessed for size. If cruraplasty is necessary, we prefer 2-0 Ethibond figure-of-eight sutures secured with the long Cor-Knot device to appropriate size.

Finally, the fundoplication is performed. Suturing is performed with 2-0 Ethibond and all sutures are secured with the long Cor-Knot device by the bedside assistant. The Mega needle driver is inserted into arm 4. The angle of His is identified, and an appropriate location on the fundus is determined for the wrap. A minimum $54 \mathrm{Fr}$ Bougie is advanced across the GEJ. The cadiere grasper is passed posterior to the GEJ and the fundus is wrapped behind it. An appropriate location for the left side of the wrap is identified by shoeshine maneuver. A suture is then passed through the left side of the wrap, then the esophagus at the GEJ, and finally the right side of the wrap, taking care to avoid injury to the well-mobilized vagus nerve. The bedside assistant grabs the needle end and draws it out. The suture should be fed from the trailing end to avoid injury to the esophagus. As the Cor-Knot device is brought in, the surgeon should approximate the fundoplication to the esophagus again to avoid undue tension. Two similar sutures are performed 1 $\mathrm{cm}$ apart proceeding distally on the GEJ, again taking care to avoid the well-mobilized vagus nerve. The bougie can be removed at this point, which facilitates cruropexy. A suture is passed through the left crus, esophagus, and stomach first and secured. Finally, a suture is passed through the right side of the wrap, the esophagus, and the right crus and secured.

\section{Postoperative care}

Patients are admitted to the stepdown floor postoperatively. They are maintained on IV fluids. A clear liquid diet is initiated in the evening on the day of surgery. Full liquids are initiated in the morning on postoperative day 1 . The patients are advanced to a soft diet prior to discharge if able, either on postoperative days 1 or 2 as clinically appropriate. A contrast esophagram is not routinely obtained, but may performed if clinically appropriate or there are any concerns about the conduct of the operation.

\section{Conclusions}

We describe our technique in performing a robot-assisted laparoscopic Nissen fundoplication using the DaVinci Xi robot platform. The main advantages of the robot-assisted approach are the magnified, stereotactic view provided by the camera, the use of bipolar energy, and the increased stability and dexterity provided by the instruments.

\section{Acknowledgments}

Funding: None.

\section{Footnote}

Provenance and Peer Review: This article was commissioned by the Guest Editor (Douglas Z Liou) for the series "Advancement in Treatment for Esophageal Diseases". This article has undergone external peer review.

Conflicts of Interest: Both authors have completed the ICMJE uniform disclosure form (available at https://jovs. amegroups.com/article/view/10.21037/jovs.2020.02.04/ coif). The series "Advancement in Treatment for Esophageal Diseases" was commissioned by the editorial office without any sponsorship or funding. DSD is a 
robotic thoracic surgery proctor for Intuitive Surgical, Inc. WBT is a robotic thoracic surgery case observation site administrator and course instructor for Intuitive Surgical, Inc. The authors have no other conflicts of interest to declare.

Ethical Statement: The authors are accountable for all aspects of the work in ensuring that questions related to the accuracy or integrity of any part of the work are appropriately investigated and resolved. All procedures performed in this study were in accordance with the Helsinki Declaration (as revised in 2013). The manuscript is waived from patient informed consent according to the ethics committee or institutional review board.

Open Access Statement: This is an Open Access article distributed in accordance with the Creative Commons Attribution-NonCommercial-NoDerivs 4.0 International License (CC BY-NC-ND 4.0), which permits the non-

doi: $10.21037 /$ jovs.2020.02.04

Cite this article as: Demos DS, Tisol WB. Robot-assisted laparoscopic Nissen fundoplication using the DaVinci Xi. J Vis Surg 2021;7:14. commercial replication and distribution of the article with the strict proviso that no changes or edits are made and the original work is properly cited (including links to both the formal publication through the relevant DOI and the license). See: https://creativecommons.org/licenses/by-nc-nd/4.0/.

\section{References}

1. Peters JH. SAGES guidelines for the management of hiatal hernia. Surg Endosc 2013;27:4407-8.

2. Yao G, Liu K, Fan Y. Robotic Nissen fundoplication for gastroesophageal reflux disease: a meta-analysis of prospective randomized controlled trials. Surg Today 2014;44:1415-23.

3. Soliman BG, Nguyen DT, Chan EY, et al. Robot-assisted hiatal hernia repair demonstrates favorable short-term outcomes compared to laparoscopic hiatal hernia repair. Surg Endosc 2020;34:2495-502. 